\title{
Integración flexible de workflows científicos en entornos de computación dinámicos
}

\author{
S. Hernández de Mesa, P. Álvarez \\ Grupo de Integración de Sistemas Distribuidos y Heterogéneos (GIDHE) \\ Instituto de Investigación en Ingeniería de Aragón (I3A) \\ Universidad de Zaragoza, Mariano Esquillor s/n, 50018, Zaragoza, Spain. \\ Tel.+34-976761000 ext. 5458, Fax+34-976762043,e-mail: shernandez@unizar.es
}

\begin{abstract}
La aparición de potentes infraestructuras de computación Grid ha impulsado un creciente interés por parte de la comunidad científica para automatizar la ejecución de sus experimentos. Para ello, la utilización de workflows científicos resulta clave de cara a aprovechar las oportunidades que presentan este tipo de infraestructuras, debido principalmente a las características que presentan: están formados por un elevado número de tareas con un alto coste computacional, que manejan grandes volúmenes de datos complejos y que requieren una gestión adecuada de los recursos tanto software como hardware disponibles. Sin embargo, la complejidad de los propios workflows y de las infraestructuras de computación provoca que el proceso de scheduling resulte complejo y difícil. Las propuestas actuales utilizan un broker de recursos que facilita esta tarea pero provoca que el diseño del workflow esté fuertemente acoplado con el middleware que gestiona la infraestructura. Esto imposibilita la utilización conjunta de diferentes Grids, la compartición y portabilidad de los workflows y la gestión flexible del ciclo de vida de los mismos. Para solucionar estas limitaciones, se plantea una infraestructura flexible a la hora de integrar workflows de diferentes lenguajes, diversas infraestructuras de computación (Grid, Cloud, etc.) y componentes de gestión que permitan enriquecer y mejorar la ejecución de los workflows. La utilización de redes de Petri de alto nivel se plantea como base, dada la amplia experiencia en este campo por parte del Grupo de Integración de Sistemas Distribuidos y Heterogéneos (GIDHE), grupo en el que se enmarca la línea de investigación del autor. Esta propuesta introduce nuevos retos en la decisión de las infraestructuras en las que ejecutar los workflows (meta-scheduling), siendo la simulación una alternativa muy adecuada al ser capaz de prever el comportamiento del workflow en diferentes escenarios.
\end{abstract}

